\title{
Design and Optimization of Nonlinear Tapers using Particle Swarm Optimization
}

\author{
Narendra Chauhan • Ankush Mittal • Dietmar Wagner • \\ M. V. Kartikeyan • M. K. Thumm
}

Received: 7 April 2008/Accepted: 24 April 2008/

Published online: 28 May 2008

(C) Springer Science + Business Media, LLC 2008

\begin{abstract}
In this paper the design and optimization of a nonlinear diameter taper, connecting the output section of a gyrotron cavity to the uniform output waveguide section, is presented. The design of a nonlinear taper of a $42 \mathrm{GHz}, 200 \mathrm{~kW} \mathrm{CW}$ gyrotron operating in the $\mathrm{TE}_{0,3}$ cavity mode with axial output collection has been taken as a case study. The taper synthesis has been carried out considering a raised cosine type of nonlinear taper and the analysis is done using a dedicated scattering matrix code. In addition, an improved particle swarm optimization - an evolutionary optimization - algorithm is used for the design optimization of this nonlinear taper. The optimum design of the taper shows the effectiveness of the presented method.
\end{abstract}

Keyword Nonlinear tapers $\cdot$ Particle swarm optimization $\cdot$ Gyrotrons

\section{Introduction}

Gyrotron output systems consist of an output diameter taper which connects the interaction region with the main waveguide system, a quasi-optical mode converter, and the RF

N. Chauhan $\cdot$ A. Mittal $\cdot$ M. V. Kartikeyan $(\bowtie)$

Department of Electronics and Computer Engineering, Indian Institute of Technology,

Roorkee 247 667, India

e-mail: kartkfec@iitr.ernet.in

D. Wagner

Max-Plank-Institut für Plasmaphysik EURATOM-IPP, Boltzmannstr. 2, 85748 Garching, Germany

M. K. Thumm

Forschungszentrum Karlsruhe, Association EURATOM-FZK,

Institut für Hochleistungsimpuls- und Mikrowellentechnik, Postfach 3640, 76021 Karlsruhe, Germany

M. K. Thumm

Universität Karlsruhe, Institut für Höchstfrequenztechnik und Elektronik,

Kaiserstraße 12, 76131 Karlsruhe, Germany

至 Springer 
window [1]. The requirement of a diameter taper in gyrotrons is to provide a good match between input and output sections of the taper with very low spurious mode content. Various taper designs such as exponential taper [2], triangular taper, Chebyshev taper [2], Klopfenstein taper [3], and Hecken taper [4] can be employed for matching purposes. Flügel and Kühn considered modified Dolph-Chebychev tapers for the analysis and design of circular waveguide tapers [5]. However, in this work, we have used a raised cosine taper profile as it yields very low mode conversion [6]. The design and optimization of the raised cosine nonlinear taper is carried out for a specific $42 \mathrm{GHz}, 200 \mathrm{~kW}$, CW gyrotron operating in the $\mathrm{TE}_{0,3}$ mode with axial output collection. In this work, the analysis of the taper was carried out using a dedicated scattering matrix code as it is very fast and accurate for taper analysis [7].

Particle Swarm Optimization (PSO) is a simple, effective and promising method used for synthesis and optimization in a multidimensional feature space. The feasibility of a simple PSO for high-power microwaves control applications is illustrated in [8]. In this paper, we presented an improved particle swarm optimization method and used it for the design optimization of this specific raised cosine type of nonlinear taper which has been considered as a case study in this work.

\section{Particle swarm optimization}

Particle swarm optimization (PSO) is a population based stochastic optimization technique developed by Eberhart and Kennedy [9]. In this algorithm, a group of potential solutions, known as particles are flown through a multidimensional search space in order to get an optimum solution. The $\mathrm{i}^{\text {th }}$ particle in the swarm (a population of particles) is represented as $\mathrm{X}_{\mathrm{i}}=\left(\mathrm{x}_{\mathrm{i} 1}, \mathrm{x}_{\mathrm{i} 2}, \ldots, \mathrm{x}_{\mathrm{iD}}\right)$, and its velocity is given by $\mathrm{V}_{\mathrm{i}}=\left(\mathrm{v}_{\mathrm{i} 1}, \mathrm{v}_{\mathrm{i} 2}, \ldots, \mathrm{v}_{\mathrm{iD}}\right)$. The best previous position of each particle is given as $\mathrm{P}_{\mathrm{i}}=\left(\mathrm{p}_{\mathrm{i} 1}, \mathrm{p}_{\mathrm{i} 2}, \ldots, \mathrm{p}_{\mathrm{iD}}\right)$. The best particle among all particles is represented by a symbol ' $g$ '. The velocity and position of each particle are updated using following relations:

$$
\begin{gathered}
v_{i d}=w^{*} v_{i d}+c_{1} * \operatorname{rand}() *\left(p_{i d}-x_{i d}\right)+c_{2} * \operatorname{rand}() *\left(p_{g d}-x_{i d}\right), \\
x_{i d}=x_{i d}+v_{i d},
\end{gathered}
$$

where $\mathrm{c}_{1}$ (cognitive constant) and $\mathrm{c}_{2}$ (social constant) are two positive constants, $\operatorname{rand}()$ is a function to generate random number between 0 and 1 , and $w$ represents the inertia weight which is used to control between local and global search.

\subsection{Parameter selection}

The ability of PSO in exploring global optima is much dependent on the choice of parameters such as $\mathrm{c}_{1}, \mathrm{c}_{2}, w$, and $\mathrm{V}_{\max }$. Various experiments for the selection of optimum parameters are demonstrated in [10]. In our experiment, a linearly decreasing inertia weight (w) from 0.95 to 0.4 , as proposed in [11], is used. The maximum velocity in each dimension is restricted to $\mathrm{V}_{\max }=\mathrm{X}_{\max }$. The value of cognitive acceleration constant $\left(\mathrm{c}_{1}\right)$ and social acceleration constant $\left(c_{2}\right)$ are fixed at 2.0 which are suggested as best in most experiments. 
2.2 Improved particle swarm optimization algorithm

An improved PSO algorithm, presented recently by the authors [12], for constrained optimization is used in this work. The algorithm is the modification of the algorithm presented by $\mathrm{Hu}$ and Eberhart [13] for handling constraints efficiently. The algorithm includes three modifications. The first one initializes particle positions and velocity within the specific range, the second one handles the constraints following a penalty based approach and the third one impose restrictions to boundary constraints by the range of parameters.

\section{Algorithm: Improved PSO for Constrained Optimization}

\section{Initialization:}

Initialize Parameters $\mathrm{c}_{1}, \mathrm{c}_{2}, \mathrm{w}$, and $\mathrm{V}_{\max }=\mathrm{X}_{\max }$

For each particle in the swarm \{

Initialize particles $(X)$ randomly in the range $\left[X_{\min }, X_{\max }\right]$

Initialize velocity of particles $(\mathrm{V})$ randomly in the range $\left[-\mathrm{V}_{\max }, \mathrm{V}_{\max }\right]$

\}

\section{Evolution:}

Do \{

For each particle \{

Calculate the fitness value and modify the fitness according to violation of constraints

If the modified fitness value is better than the best fitness value (pBest) in history, set current value as the new pBest.

\}

Choose the particle with the best fitness value of all the particles as the gBest For each particle \{

Calculate particle velocity according to equation (1a)

Restrict the velocity of particles by $\left[-V_{\max }, V_{\max }\right]$

Update particle's position according to equation (1b)

Restrict the particle's position by $\left[\mathbf{X}_{\min }, \mathbf{X}_{\max }\right]$

\}

\} While maximum iteration or minimum error criteria is not attained

Fig. 1 Improved PSO algorithm for constrained optimization [12].

Springer 
Table 1 Range of design parameters.

\begin{tabular}{ll}
\hline Design parameter & Range \\
\hline Length $(\mathrm{L})$ & $200-350 \mathrm{~mm}$ \\
Radius at output end $\left(\mathrm{r}_{2}\right)$ & $35-45 \mathrm{~mm}$ \\
Number of sections $(\mathrm{N})$ & $50-500$ \\
Gamma $(\gamma)$ & $0.1-1.0$ \\
\hline
\end{tabular}

The advantage of the proposed modifications is that the initialized particles need not to satisfy all the constraints as it is required in the algorithm [13], thus making the algorithm easy to work and efficient to handle design constraints. The proposed algorithm is as shown in Fig. 1 with the modifications highlighted.

\section{Experiment}

In this paper, the design of a nonlinear taper of raised cosine type has been performed following Wagner [14] for a $42 \mathrm{GHz}, 200 \mathrm{~kW}$, CW gyrotron. In this context, we considered four nominal parameters of the nonlinear taper namely - the length of the taper (L), the radius of the taper at the output end $\left(\mathrm{r}_{2}\right)$, the number of sections to be discredited $(\mathrm{N})$, and a geometrical parameter gamma $(\gamma)$ while keeping the radius of the taper at input end $\left(\mathrm{r}_{1}\right)$ constant. The range of design parameters considered for the design are summarized in Table 1. The objective for the design was to obtain a maximum transmission coefficient (i.e. $\mathrm{S}_{21}$-parameter), operating with $\mathrm{TE}_{0,3}$ mode with minimized spurious mode content.

The optimization of the design parameters for each taper was carried out using the improved PSO algorithm as presented in the previous section. The constraints considered for the problem were the boundary constraints imposed by the range of each parameter as specified in Table 1 . The algorithm was executed with swarm sizes 5, and 10, respectively, for about 100 iterations in each case. The iterations considered for the design were sufficient for the convergence of the swarm. The convergence graph of improved PSO for each swarm size is shown in Fig. 2.

Fig. 2 Convergence of improved PSO.

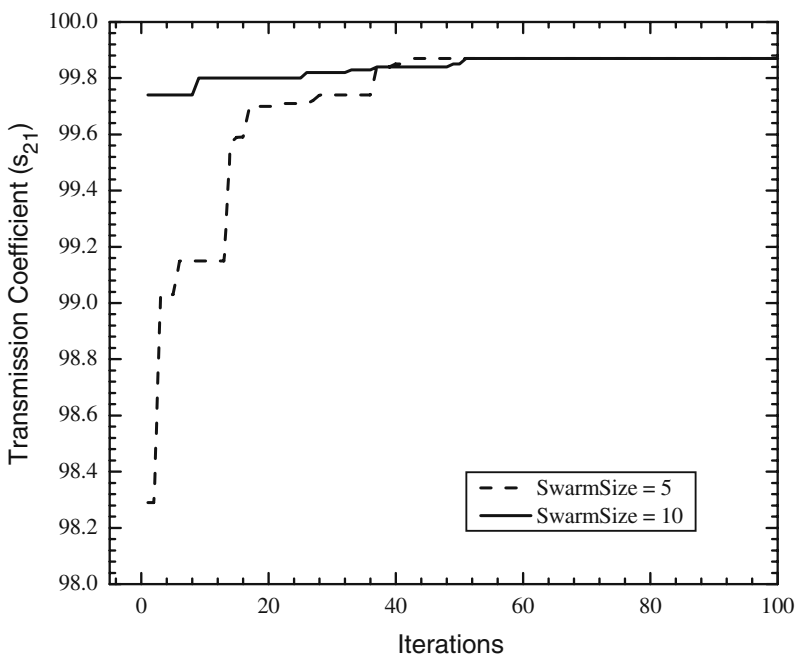


Fig. 3 Effect of gamma parameter $(\gamma)$ on the synthesis of raised cosine taper $\left(\mathrm{L}=350 \mathrm{~mm}, \mathrm{r}_{2}=\right.$ $35.0 \mathrm{~mm}, \mathrm{~N}=466$ ).

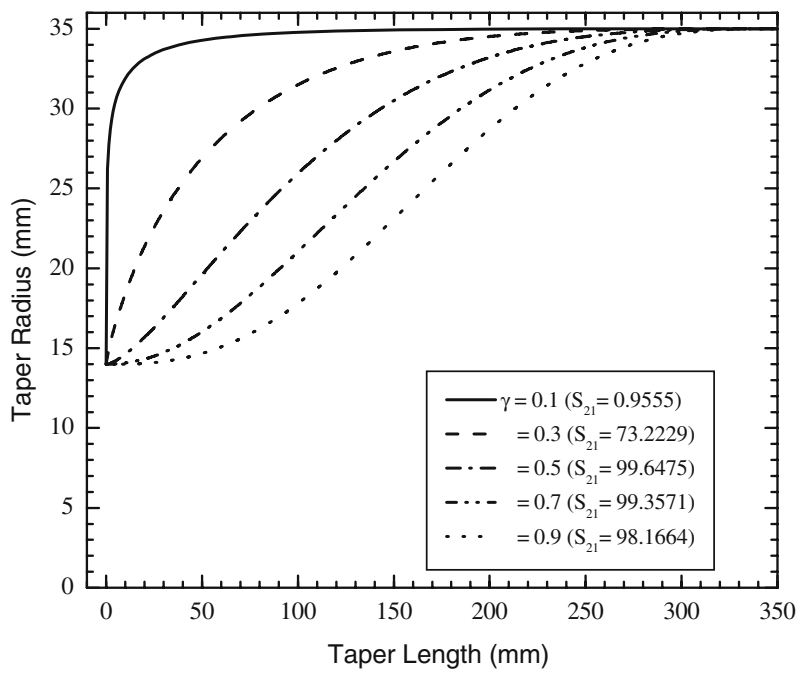

A transmission of $99.86 \%$ for the operating $\mathrm{TE}_{0,3}$ mode was obtained using optimized design parameters. The optimized taper has a length of $349.99 \mathrm{~mm}$, an output radius of $37.32 \mathrm{~mm}$, consists of 208 sections, and has a value of the gamma parameter of 0.504 . According to our experiments, we found that other combinations of optimum parameters are also possible to get similar transmission coefficient. For instance, we also obtained a transmission of $99.85 \%$ for the same operating mode $\left(\mathrm{TE}_{0,3}\right)$ using $\mathrm{L}=315.45 \mathrm{~mm}, \mathrm{r}_{2}=$ $35.0 \mathrm{~mm}, \mathrm{~N}=469$, and $\gamma=0.517$. Multiple solutions are possible in different runs of the PSO algorithm due to its stochastic nature. We have also observed the effects of varying gamma parameter $(\gamma)$, radius at the output end of the taper $\left(r_{2}\right)$, and length of the taper $(L)$ on the taper synthesis and the transmission coefficient. The effect of the gamma parameter

Fig. 4 Effect of radius $\left(r_{2}\right)$ on the synthesis of raised cosine taper $(\mathrm{L}=350, \gamma=0.5, \mathrm{~N}=466)$.

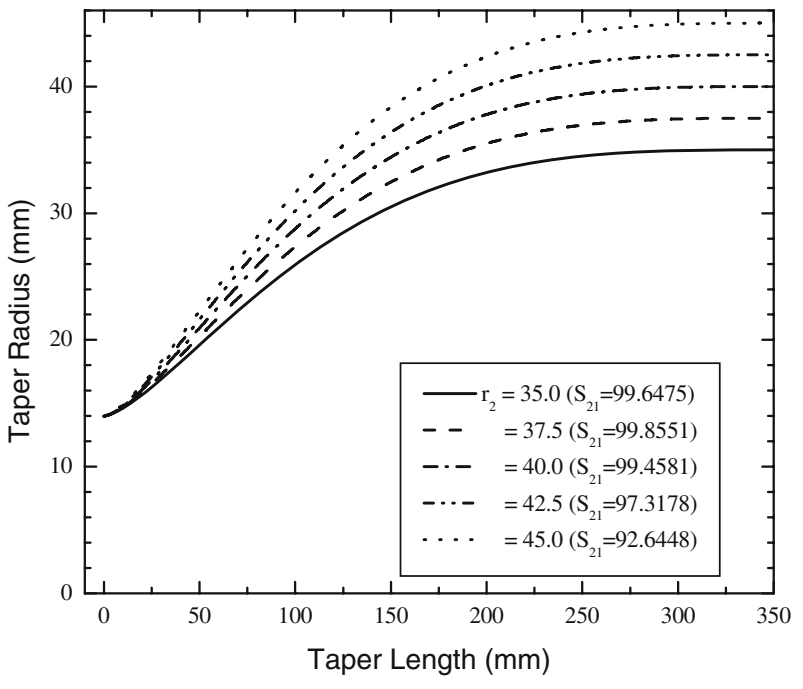


Fig. 5 Effect of length (L) on the synthesis of raised cosine taper $\left(\mathrm{r}_{2}=35 \mathrm{~mm}, \gamma=0.5, \mathrm{~N}=466\right)$.

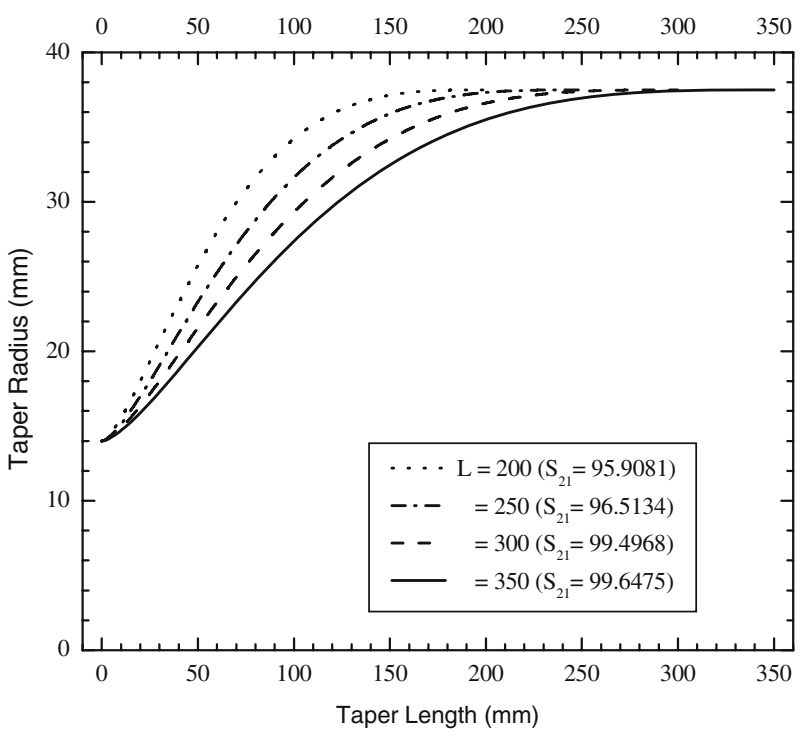

is shown in Fig. 3, while the effect of radius at output end of the taper on the transmission coefficient is shown in Fig. 4.

The percentage of transmission for the main mode $\left(\mathrm{TE}_{0,3}\right)$ for various taper lengths within the desired range are shown in Fig. 5. In addition, the percentage of transmission and reflection of the main mode along with its azimuthal neighbours for an optimized run (with $\mathrm{r}_{1}=13.991, \mathrm{r}_{2}=37.32, \mathrm{~L}=349.99, \gamma=0.504, \mathrm{~N}=208$ ) are shown in Table 2.

\section{Conclusions}

An improved particle swarm optimization algorithm [12] is used for the design of a raised cosine non-linear taper for a specific high power gyrotron. A dedicated scattering matrix code was used for the fast and accurate analysis of the taper during the design process. The selection of PSO parameters was carried out in accordance with previous experimental investigations. The optimized results show the best matching obtained with the raised cosine taper which confirms effectiveness of this method for the design of nonlinear tapers.

Table 2 Percentage of transmitted and reflected power (Frequency: $42.0 \mathrm{GHz}$ ).

\begin{tabular}{lll}
\hline mode & Reflection [\%] & Transmission [\%] \\
\hline TE $_{0,1}$ & 0.00001 & 0.01240 \\
TE $_{0,2}$ & 0.00010 & 0.02046 \\
TE $_{0,3}$ & 0.00051 & 99.86208 \\
TE $_{0,4}$ & 0.01176 & 0.00240 \\
TE $_{0,5}$ & 0.00126 & 0.00032 \\
TE $_{0,6}$ & 0.00038 & 0.00009 \\
TE $_{0,7}$ & 0.00016 & 0.00002 \\
TE $_{0,8}$ & 0.00008 & 0.00021 \\
TE $_{0,9}$ & 0.00004 & 0.00007 \\
TE $_{0,10}$ & 0.00003 & 0.00002 \\
\hline
\end{tabular}


The time required for the optimization was 25 minutes approximately on a dual core processor. Though the scope of this work is limited to the design optimization of a raised cosine tapers for high power gyrotrons, one may extend this treatment for the design optimization of other types of nonlinear tapers as well.

Acknowledgements One of the authors (MVK) thank the Department of Science and Technology, Govt. of India, for their generous support in the form of a grant-in-aid project. The authors thank Mr. Arun Kumar and Ms. S. Kamakshi for their help and assistance in the preparation of necessary design data.

\section{References}

1. M. V. Kartikeyan, E. Borie, and M. Thumm, Gyrotrons - High Power Microwave And Millimeter Wave Technology, (Springer-Verlag, Berlin-Heidelberg, Germany, 2004).

2. R. E. Collin, Foundations for Microwave Engineering, 2nd Ed. (McGraw-Hill, New York, 1992), pp. $370-386$.

3. R. W. Klopfenstein, A Transmission Line Taper of Improved Design, Proceedings of the IRE, 44, p. 3135 , (1956), January.

4. R. P. Hecken, A Near-Optimum Matching Section Without Discontinuities, IEEE Trans. Microwave Theor. Tech., 20(11), 734-739, (1972), November.

5. H. Flügel, and E. Kühn, Computer-aided analysis and design of circular waveguide tapers, IEEE Trans. Microwave Theor. Tech., 36(2), 332-336, (1988), February.

6. W. G. Lawson, Theoretical evaluation of non-linear tapers for a high power gyrotrons, IEEE Trans. Microwave Theor. Tech., 38(11), 1617-1622, (1990).

7. D. Wagner, M. Thumm, G. Gantenbein, W. Kasperek, and T. Idehara, Analysis of a complete gyrotrons oscillator using the scattering matrix description, Int. J. Infrared Millimeter Waves, 19(2), 185-194, (1998).

8. A. A. Bogdashov, and Y. V. Rodin, Mode Converter Synthesis by the Particle Swarm Optimization, Int. J. Infrared Millimeter Waves, 28, 627-638, (2007).

9. J. Kennedy, and R. Eberhart, Particle swarm optimization, Proc. 4th IEEE Int. Conf. on Neural Networks, 1942-1948, (1995).

10. Y. Shi, and R. Eberhart, Parameter selection in particle swarm optimization, Proc. of 7th Annual Conference on Evolution Computation, 591-601, (1998).

11. Y. Shi, and R. Eberhart, Empirical study of particle swarm optimization, Proc. 1999 Congress on Evolutionary Computation, 1945-1950, (1999).

12. N. Chauhan, K. Roy, A. Kumar, A. Mittal, M.V. Kartikeyan, SVM-PSO based modeling and optimization of microwave components, FREQUENZ - J. RF Engineering and Telecommunications, no. $1-2,18-24,(2008)$.

13. $\mathrm{X}$. $\mathrm{Hu}$, and R. Eberhart, Solving constrained nonlinear optimization problems with particle swarm optimization, Proc. 6th World Multiconference on Systemics, Cybernetics and Informatics, (2002).

14. D. Wagner, W. Kasparek, M. Thumm, Optimized nonlinear cavity and uptaper for gyrotron FU V, 11th Joint German Russian Meeting on ECRH and Gyrotrons, (1999), June 23-29. 